\title{
Rationality of the moduli spaces of plane curves of sufficiently large degree
}

\author{
Christian Böhning • Hans-Christian Graf von Bothmer
}

Received: 26 May 2008 / Accepted: 17 July 2009 / Published online: 19 August 2009

(C) The Author(s) 2009. This article is published with open access at Springerlink.com

\begin{abstract}
We prove that the moduli space of plane curves of degree $d$ is rational for all sufficiently large $d$.
\end{abstract}

\section{Introduction}

It is a classical question, which can be traced back to works of Hilbert and Emmy Noether, whether the orbit spaces $\mathbb{P} / G$ are rational where $\mathbb{P}$ is a projective space and $G$ is a reductive algebraic group acting linearly in $\mathbb{P}$. If $G$ is not assumed connected, in fact for $G$ a finite solvable group, D. Saltman has shown in [21] that the answer to this question is negative in general (Emmy Noether had apparently conjectured that the quotient should be rational in this case). No counterexamples are known for connected complex reductive groups $G$.

For simply connected classical groups except $\operatorname{Spin}_{n}(\mathbb{C})$ for $n>12$, the quotients $\mathbb{P} / G$ are known to be stably rational, cf. [4, 9]. Bogomolov [4] claims the result for all $\operatorname{Spin}_{n}(\mathbb{C})$ but the proof contains a mistake pointed out by P. Katsylo. The question whether stably rational varieties are always rational is the well-known Zariski problem which Beauville, Colliot-Thélène, Sansuc and Swinnerton-Dyer [2] answered in the negative as well: There are three-dimensional conic bundles $X$ over rational surfaces which are irrational, but $X \times \mathbb{P}^{3}$ is rational. This uses the method of intermediate Jacobians by Clemens-Griffiths [8] which, however, seems to work only for threefolds. In general, it is rather hard to distinguish stably rational and rational varieties. The method connected with the birational invariance of the Brauer-Grothendieck group used previously by Artin and Mumford [1] to obtain more elementary examples of unirational non-rational threefolds, is insensitive to this distinction

Both authors were supported by the German Research Foundation (Deutsche Forschungsgemeinschaft (DFG)) through the Institutional Strategy of the University of Göttingen.

C. Böhning $(\bowtie) \cdot$ H.-C. Graf von Bothmer

Mathematisches Institut, Georg-August-Universität Göttingen, Bunsenstr. 3-5, 37073 Göttingen,

Germany

e-mail: boehning@uni-math.gwdg.de

H.-C. Graf von Bothmer

e-mail: bothmer@uni-math.gwdg.de 
(unirational varieties constitute a strictly bigger class than stably rational ones; e.g. Saltman's counterexamples mentioned above are not even stably rational). The reader may find these and other methods to prove irrationality, including the use of Noether-Fano inequalities via untwisting of birational maps and Kollár's method of differential forms in characteristic $p$ to prove non-rationality of some general hypersurfaces, in the survey by V.A. Iskovskikh and Yu.G. Prokhorov [15].

The geometrically most relevant case of the general question discussed above seems to be the case of the moduli space of projective hypersurfaces of degree $d$ in $\mathbb{P}^{n}$, which we denote by $\operatorname{Hyp}(d, n)$. Here rationality is known in the following cases:

- $n=1$ (the classical case of binary forms resp. sets of points on the projective line), $d$ odd [16], $d$ even [5, 6]

- $n=2, d \leq 3$ (well known), $d=4([18,19]), d \equiv 1(\bmod 4)([22]), d \equiv 1(\bmod 9)$ and $d \geq 19$ [22]; $d \equiv 0(\bmod 3)$ and $d \geq 1821$ [17] (this article contains the remark that the author obtained the result also for $d \geq 210$, unpublished); the same paper also gives some results for congruences to the modulus 39; furthermore, there are some unpublished additional cases in the case of plane curves which we do not try to enumerate.

- $n=3, d \leq 2$ (obvious), $d=3$ (Clebsch and Salmon; but see [3]).

- $n>3, d \leq 2$ (obvious).

This represents what we could extract from the literature. It is hard to say if it is exhaustive. The reader may consult the very good (though not recent) survey article [10] for much more information on the rationality problem for fields of invariants.

The main theorem of the present article is:

Theorem 1.1 The moduli space of plane curves of sufficiently large degree $d \gg 0$ under projective equivalence is rational.

More precisely, for $d=3 n, d \geq 1821$, this was proven by Katsylo [17] as a glance back at the preceding summary shows. We use this result and don't improve the bound for $d$. For $d \equiv 1(\bmod 3)$, we obtain rationality for $d \geq 37$. For $d \equiv 2(\bmod 3)$, we need $d \geq 65$.

Let us turn to some open problems. First of all, the method used in this paper seems to generalize and -provided the required genericity properties hold and can be verified computationally- could yield a proof of the rationality of $\operatorname{Hyp}(d, n)$ for fixed $n$ if the degree $d$ is large enough and $n+1$ does not divide $d$. The latter case might be amenable to the techniques of [17] in general. Thus the case of the moduli spaces of surfaces of degree $d$ in $\mathbb{P}^{3}$ seems now tractable with some diligence and effort. But we do not see how one could obtain results on $\operatorname{Hyp}(d, n)$ for all $n$ (and $d$ sufficiently large compared to $n$ ).

More importantly, whereas we think that it is highly plausible that $\operatorname{Hyp}(d, n)$ is always rational if $d$ is sufficiently large compared to $n$, we do not want to hazard any guess in the case where $d$ is small. In fact, we do not know any truly convincing philosophical reason why $\operatorname{Hyp}(d, n)$ should be rational in general; the present techniques of proving rationality always seem to force one into assuming that $d$ is sufficiently large if one wants to obtain an infinite series of rational examples by a uniform method. Moreover, it can be quite painstaking and tricky to get a hold of the situation if $d$ is small as Katsylo's tour de force proof for $\mathfrak{M}_{3}$ (i.e. Hyp $(4,2)$ ) in $[18,19]$ amply illustrates. The maybe easiest unsolved cases are $\operatorname{Hyp}(6,2)$ (plane sextics) and $\operatorname{Hyp}(4,3)$ (quartic surfaces). Note that the former space is birational to the moduli space of polarized K3 surfaces $(S, h)$ of degree 2 (thus $S$ is a nonsingular projective $\mathrm{K} 3$ surface and $h \in \operatorname{Pic}(S)$ is the class of an ample divisor with $h^{2}=2$ ), and the latter space is birational to the moduli space of polarized K3 surfaces of degree 4 . 


\section{Outline of proof}

The structural pattern of the proof is similar to [22]; there the so called method of covariants is introduced, and we learnt a lot from studying that source.

We fiber the space $\mathbb{P}\left(\operatorname{Sym}^{d}\left(\mathbb{C}^{3}\right)^{\vee}\right)$ of degree $d$ plane curves over the space of plane quartics, if $d \equiv 1(\bmod 3)$, and over the space of plane octics, if $d \equiv 2(\bmod 3)$, i.e. we construct $\mathrm{SL}_{3}(\mathbb{C})$-equivariant maps

$$
S_{d}: \operatorname{Sym}^{d}\left(\mathbb{C}^{3}\right)^{\vee} \rightarrow \operatorname{Sym}^{4}\left(\mathbb{C}^{3}\right)^{\vee}
$$

and

$$
T_{d}: \operatorname{Sym}^{d}\left(\mathbb{C}^{3}\right)^{\vee} \rightarrow \operatorname{Sym}^{8}\left(\mathbb{C}^{3}\right)^{\vee}
$$

( $S_{d}$ coincides with the covariant used in [22] for the case $\left.d=9 n+1\right)$. These maps are of degree 4 as polynomials in the coordinates on the source spaces, i.e. of degree 4 in the curve coefficients. They are constructed via the symbolic method recalled in section 3. Furthermore they induce dominant rational maps on the associated projective spaces. We remark here that the properties of $S_{d}$ and $T_{d}$ essential for the proof are that they are of fixed low degree in the curve coefficients, take values in spaces of curves of fixed low degree, and are sufficiently generic.

We now focus on the case $d \equiv 1(\bmod 3)$. The proof has three main steps:

(1) $\operatorname{Hyp}(4,2)$ is stably rational, more precisely its product with $\mathbb{P}^{8}$ is rational; cf. [6], Theorem 1.1 for this.

(2) We find a linear subspace $L_{S} \subset \operatorname{Sym}^{d}\left(\mathbb{C}^{3}\right)^{\vee}$ such that $\mathbb{P}\left(L_{S}\right)$ is contained in the base locus $B_{S_{d}}$ of $S_{d}$ with a full triple structure, i.e. $I_{\mathbb{P}\left(L_{S}\right)}^{3} \supset I_{B_{S_{d}}}$, and consider the projection $\pi_{L_{S}}$ away from $\mathbb{P}\left(L_{S}\right)$ onto $\mathbb{P}\left(\operatorname{Sym}^{d}\left(\mathbb{C}^{3}\right)^{\vee} / L_{S}\right)$. We show that a general fibre of $S_{d}$ is birationally a vector bundle over a rational base.

(3) The quotient map

$$
\operatorname{PSym}^{4}\left(\mathbb{C}^{3}\right)^{\vee} \rightarrow\left(\mathbb{P S y m}^{4}\left(\mathbb{C}^{3}\right)^{\vee}\right) / \mathrm{PGL}_{3}(\mathbb{C})
$$

has a section $\sigma_{4}$. Pulling back the linear fibrations constructed in (2) via $\sigma_{4}$ we show that the moduli space of plane curves of degree $d$ is birational to $\operatorname{Hyp}(4,2) \times \mathbb{P}^{N}$, where $N$ is large, whence we conclude by (1).

The main computational difficulty occurs in (2) where we have to establish that $L_{S}$ is sufficiently generic. Projecting from $\mathbb{P}\left(L_{S}\right)$ we obtain a diagram

$$
\begin{gathered}
\operatorname{PSym}^{d}\left(\mathbb{C}^{3}\right)^{\vee}--S_{d} \\
\vdots \\
\checkmark \pi_{L_{S}} \\
\vee \\
\mathbb{P}\left(\operatorname{Pym}^{d}\left(\mathbb{C}^{3}\right)^{\vee} / L_{S}\right)
\end{gathered}
$$

We show that

(*) for a particular (hence a general) $\bar{g} \in \mathbb{P}\left(\operatorname{Sym}^{d}\left(\mathbb{C}^{3}\right)^{\vee} / L_{S}\right)$ the map

$$
\left.S_{d}\right|_{\mathbb{P}\left(L_{S}+\mathbb{C} g\right)}: \mathbb{P}\left(L_{S}+\mathbb{C} g\right)-\rightarrow \mathbb{P S y m}{ }^{4}\left(\mathbb{C}^{3}\right)^{\vee}
$$

is surjective. 
Fig. 1 A non-generic $L$

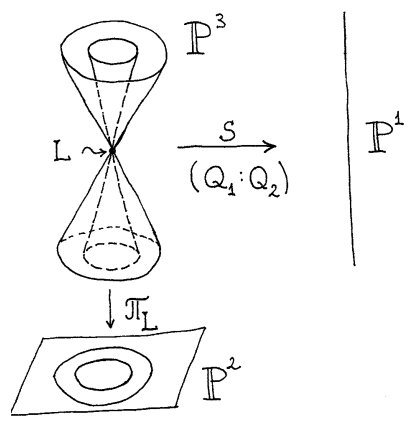

Note that $\left.S_{d}\right|_{\mathbb{P}\left(L_{S}+\mathbb{C g}\right)}$ is linear since $L_{S}$ is contained in the base locus with full triple structure and $S_{d}$ is of degree 4 in the curve coefficients. It is therefore enough to explicitly construct points in the image that span $\operatorname{PSym}^{4}\left(\mathbb{C}^{3}\right)^{\vee}$. From this it follows at once that a general fibre of $S_{d}$ is mapped dominantly by $\pi_{L_{S}}$ whence we may view such a fibre birationally as a vector bundle over a rational base. To understand better why the dominance of $S_{d}$ is not sufficient here, it is instructive to keep the following example in mind:

Example 1 Consider the rational map

$$
S: \quad \mathbb{P}^{3} \rightarrow \mathbb{P}^{1}, \quad x \mapsto\left(Q_{1}(x): Q_{2}(x)\right)
$$

where $Q_{1}, Q_{2}$ are quadric cones with vertex $L . S$ is dominant. Projection from the vertex $L$ to $\mathbb{P}^{2}$ is also dominant, but the quadric cones (i.e. the fibers of $S$ ) do not map dominantly to $\mathbb{P}^{2}$ (see Fig. 1). The projection fibers are the lines through $L$ and indeed each such line is contained completely in one cone in the pencil $\lambda Q_{1}+\mu Q_{2}$.

The base locus $B$ of $S$ consists of 4 lines that meet in $L$. If on the other hand we project from a smooth point $L^{\prime} \subset B$ then a general fiber of $S$ maps dominantly to $\mathbb{P}^{2}$. Indeed a general line through $L^{\prime}$ intersects all cones.

The complications in proving $(*)$ arise due to the fact that the natural description of $L_{S}$ is in terms of the monomials which span it, whereas $S_{d}$ can be most easily evaluated on forms which are written as sums of powers of linear forms, see for example [20]. These two points of view do not match, and we cannot repose on methods in [22]. Instead we introduce new techniques in Sect. 5 to solve this difficulty:

- We use interpolation polynomials to write down elements in $L_{S}$ as sums of powers of linear forms.

- Next we employ considerations of leading terms (or, geometrically, jets at infinity) to eliminate the interpolation polynomials, from our formulae.

- For large enough $d=3 n+1$, we finally reduce $(*)$ to the property that a certain matrix $M(n)$ has full rank. The size of $M(n)$ is independent of $n$ while (and this is the main point) its entries are of the form

$$
\sum_{v} \rho_{v}^{n} P_{v}(n)
$$

where $P_{v}$ are polynomials of fixed degree (i.e. independent of $n$ ), $\rho_{v}$ are constants, and the number of summands in the expression is independent of $n$. This is possible only because we eliminated the interpolation polynomials in the previous step. 
- By choosing a point $g$ with integer coefficients we can arrange that $\rho_{v}$ and $P_{v}(n)$ are defined over $\mathbb{Q}$ with denominators that are not divisible by a small prime $\wp$ which we call the precision of our calculation. Thus if we work over the finite field $\mathbb{F}_{\wp}$, the matrix $M(n)$ is periodic in $n$ with period $\wp(\wp-1)$. A computer algebra program is then used to check that these matrices all have full rank. By semicontinuity, this proves that $M(n)$ has full rank for all $n$ in characteristic 0 .

In a rather round-about sense, we have also been guided by the principle that evaluation of a polynomial at a special point can be much cheaper than computing the polynomial.

\section{Notation and definition of the covariants}

For definiteness, the base field will be $\mathbb{C}$, the field of complex numbers, though one might replace it by any (not necessarily algebraically closed) field of characteristic 0 throughout.

Let $G:=\mathrm{SL}_{3}(\mathbb{C})$, and let $\bar{G}:=\mathrm{PGL}_{3}(\mathbb{C})$ be the adjoint form of $G$. We denote by $V(k)$ the irreducible $G$-representation $\operatorname{Sym}^{k}\left(\mathbb{C}^{3}\right)^{\vee}$. We fix a positive integer $d$ not divisible by 3 , $d=3 n+1$ or $d=3 n+2, n \in \mathbb{N}$.

The symbol $[k], k \in \mathbb{N}$, denotes the set of integers from 0 (incl.) to $k$ (incl.). Let $x_{1}, x_{2}, x_{3} \in\left(\mathbb{C}^{3}\right)^{\vee}$ denote the basis dual to the standard basis in $\mathbb{C}^{3}$ and put $\mathbf{x}:=\left(x_{1}, x_{2}, x_{3}\right)$. We will use Schwartz's multi-index notation and denote multi-indices by lower case boldface letters. Thus we write a general homogeneous form $f \in V(d)$ of degree $d$ as

$$
f=\sum_{\mathbf{i} \in[d]^{3}, \mathbf{i} \mid=d} \frac{d !}{\mathbf{i} !} A_{\mathbf{i}} \mathbf{x}^{\mathbf{i}},
$$

where $\mathbf{i} !=i_{1} ! i_{2} ! i_{3} !,|\mathbf{i}|:=i_{1}+i_{2}+i_{3}, A_{\mathbf{i}}=A_{i_{1} i_{2} i_{3}}, \mathbf{x}^{\mathbf{i}}=x_{1}^{i_{1}} x_{2}^{i_{2}} x_{3}^{i_{3}}$. We will use the symbolical method introduced by Aronhold and Clebsch to write down $G$-equivariant maps (covariants) from $V(d)$ to $V(4)$ (if $d=3 n+1$ ) or to $V(8)$ (if $d=3 n+2$ ). It is explained in [14] and, from a modern point of view, in [11], Chap. 1 . We denote by $\alpha=\left(\alpha_{1}, \alpha_{2}, \alpha_{3}\right)$ a vector of symbolic variables, and also introduce vectors $\beta, \gamma, \delta$, similarly. We write $\alpha_{\mathbf{x}}=\alpha_{1} x_{1}+\alpha_{2} x_{2}+\alpha_{3} x_{3}$, and similarly $\beta_{\mathbf{x}}, \gamma_{\mathbf{x}}, \delta_{\mathbf{x}}$. Moreover we define the bracket factor $(\alpha \beta \gamma)$ by

$$
(\alpha \beta \gamma):=\operatorname{det}\left(\begin{array}{lll}
\alpha_{1} & \alpha_{2} & \alpha_{3} \\
\beta_{1} & \beta_{2} & \beta_{3} \\
\gamma_{1} & \gamma_{2} & \gamma_{3}
\end{array}\right)
$$

and write $(\alpha \beta \delta)$ etc. similarly. The idea in this calculus is to write $f \in V(d)$ symbolically as a power of a linear form in several ways:

$$
f=\alpha_{\mathbf{x}}^{d}=\beta_{\mathbf{x}}^{d}=\gamma_{\mathbf{x}}^{d}=\delta_{\mathbf{x}}^{d},
$$

whence the identities

$$
A_{\mathbf{i}}=\alpha^{\mathbf{i}}=\beta^{\mathbf{i}}=\gamma^{\mathbf{i}}=\delta^{\mathbf{i}}
$$

If $d=3 n+1$, define a covariant $S_{d}: V(d) \rightarrow V(4)$ of order 4 and degree 4 by the following prescription:

$$
\begin{aligned}
I(\alpha, \beta, \gamma, \delta) & :=(\alpha \beta \gamma)(\alpha \beta \delta)(\alpha \gamma \delta)(\beta \gamma \delta), \\
S_{d}(\alpha, \beta, \gamma, \delta) & :=I^{n} \alpha_{\mathbf{x}} \beta_{\mathbf{x}} \gamma_{\mathbf{x}} \delta_{\mathbf{x}} .
\end{aligned}
$$


The formula for $S_{d}$ should be read in the following way: The right-hand side of (5), when we multiply it out formally, is a sum of monomials $\alpha^{\mathbf{i}} \beta^{\mathbf{j}} \gamma^{\mathbf{k}} \delta^{\mathbf{l}} \mathbf{x}^{\mathbf{e}}, \mathbf{i}, \mathbf{j}, \mathbf{k}, \mathbf{l} \in[d]^{3}, \mathbf{e} \in[4]^{3}$, and $|\mathbf{i}|=\cdots=|\mathbf{I}|=d,|\mathbf{e}|=4$. Thus one can use (1) and (3) to rewrite the right-hand side unambiguously in terms of the coefficients $A_{\mathbf{i}}$ of $f \in V(d)$. Hence $S_{d}$ may be viewed as a map from $V(d)$ to $V(4)$, homogeneous of degree 4 in the coefficients $A_{\mathbf{i}}$, which is clearly $G$-equivariant. By abuse of notation, we denote the induced rational map

$$
S_{d}: \quad \mathbb{P} V(d) \rightarrow \mathbb{P} V(4)
$$

by the same letter. Note that $I$ defined by (4) may be viewed as an invariant of plane cubics $I: V(3) \rightarrow \mathbb{C}$ of degree 4 in the coefficients of the cubic. In fact, this is the famous Clebsch invariant, vanishing on the locus of Fermat cubics, or vanishing on the equi-anharmonic cubics, i.e. nonsingular plane cubics which can be written as a double cover of $\mathbb{P}^{1}$ branched in four points with equi-anharmonic cross-ratio. Equi-anharmonic cross-ratio means crossratio equal to minus a cube root of 1 . Equi-anharmonic quadruples of points in $\mathbb{P}^{1}$ are one of the two possible $\mathrm{PGL}_{2} \mathbb{C}$-orbits of 4 points in $\mathbb{P}^{1}$ with non-trivial isotropy group (the other orbit being quadruples with harmonic cross-ratio, i.e. equal to $-1,1 / 2$ or 2 ). See (5.13) of [13], for details.

The letter $S$ in $S_{d}$ was chosen in honor of the 19th century Italian geometer Gaetano Scorza, who studied in detail the map $S_{4}$, called the Scorza map (cf. [13], Sects. 6 and 7 , and [12], Sect. 6.4.1).

Similarly, for $d=3 n+2$, we define a covariant $T_{d}: V(d) \rightarrow V(8)$ of order 8 and degree 4 by

$$
T_{d}(\alpha, \beta, \gamma, \delta):=I^{n} \alpha_{\mathbf{x}}^{2} \beta_{\mathbf{x}}^{2} \gamma_{\mathbf{x}}^{2} \delta_{\mathbf{x}}^{2} .
$$

and denote the induced rational map $T_{d}: \mathbb{P} V(d) \rightarrow \mathbb{P} V(8)$ by the same letter.

We remark that it is hard to calculate the values of $S_{d}$ (or $T_{d}$ ) on a general homogeneous form $f$ of degree $d$ without knowing the entire expression of $S_{d}$ (resp. $T_{d}$ ) as a polynomial in the coefficients $A_{\mathbf{i}}$, which is awkward. One can, however, work directly with the symbolic expressions given in (5) and (7) if one writes $f$ as a linear combination of $d$-th powers of linear forms:

$$
f=\lambda_{1} l_{1}^{d}+\cdots+\lambda_{N} l_{N}^{d}, \quad \text { some } N \in \mathbb{N} .
$$

For linear forms $l_{i}, l_{j}, l_{k}, l_{p} \in \mathbb{C}\left[x_{1}, x_{2}, x_{3}\right]_{1}$ we use the notation

$$
I\left(l_{i}, l_{j}, l_{k}, l_{p}\right), \quad S_{d}\left(l_{i}, l_{j}, l_{k}, l_{p}\right), \quad T_{d}\left(l_{i}, l_{j}, l_{k}, l_{p}\right)
$$

which is defined via formulas (4), (5), (7), but where for the vectors $\alpha, \beta, \gamma, \delta$ of symbolic variables we substitute the vectors of coordinates w.r.t. $x_{1}, x_{2}, x_{3}$ of $l_{i}, l_{j}, l_{k}$, and $l_{p}$. One then has the following easy, but fundamental multi-linearity properties of $S_{d}$ and $T_{d}$ whose proof is a straight-forward computation and therefore omitted.

Lemma 3.1 We have

$$
\begin{aligned}
& S_{d}(f)=24 \sum \lambda_{i} \lambda_{j} \lambda_{k} \lambda_{p} S_{d}\left(l_{i}, l_{j}, l_{k}, l_{p}\right), \\
& T_{d}(f)=24 \sum \lambda_{i} \lambda_{j} \lambda_{k} \lambda_{p} T_{d}\left(l_{i}, l_{j}, l_{k}, l_{p}\right) .
\end{aligned}
$$

The right-hand sums run over all $(i, j, k, p)$ with $1 \leq i<j<k<p \leq N$. 


\section{Special linear subspaces of the base loci}

The group $G=\mathrm{SL}_{3} \mathbb{C}$ is a rank 2 complex semisimple algebraic group, and choosing the standard torus $T$ of diagonal matrices as maximal torus, and the group of upper-triangular matrices as Borel subgroup, one has the notions of roots, positive and simple roots, and simple coroots $H_{1}, H_{2}$ available. Corresponding to $H_{1}, H_{2}$ one has one-parameter subgroups $\lambda_{H_{1}}, \lambda_{H_{2}}: \mathbb{C}^{*} \rightarrow T$ given by

$$
\lambda_{H_{1}}(t)=\left(\begin{array}{ccc}
t & 0 & 0 \\
0 & t^{-1} & 0 \\
0 & 0 & 1
\end{array}\right), \quad \lambda_{H_{2}}(t)=\left(\begin{array}{ccc}
1 & 0 & 0 \\
0 & t & 0 \\
0 & 0 & t^{-1}
\end{array}\right) .
$$

For $d=3 n+1$, we may view the covariant $S_{d}$ as an element in

$$
\left(\operatorname{Sym}^{4} V(d)^{\vee} \otimes V(4)\right)^{G},
$$

a $G$-invariant polynomial of degree 4 in the curve coefficients $A_{\mathbf{i}}, \mathbf{i} \in[d]^{3},|\mathbf{i}|=d$, with values in $V(4)$. As such it is a linear combination of monomials

$$
A_{\mathbf{i}} A_{\mathbf{j}} A_{\mathbf{k}} A_{\mathbf{l}} \mathbf{x}^{\mathbf{e}},
$$

where $\mathbf{i}, \mathbf{j}, \mathbf{k}, \mathbf{l} \in[d]^{3},|\mathbf{i}|=\cdots=|\mathbf{k}|=d, \mathbf{e} \in[4]^{3},|\mathbf{e}|=4$.

Similarly, for $d=3 n+2, T_{d}$ can be viewed as an element of

$$
\left(\operatorname{Sym}^{4} V(d)^{\vee} \otimes V(8)\right)^{G},
$$

i.e. a $G$-invariant polynomial of degree 4 in the curve coefficients $A_{\mathbf{i}}, \mathbf{i} \in[d]^{3},|\mathbf{i}|=d$, with values in $V(8)$. It is a linear combination of monomials

$$
A_{\mathbf{i}} A_{\mathbf{j}} A_{\mathbf{k}} A_{\mathbf{l}} \mathbf{x}^{\mathbf{e}},
$$

where $\mathbf{i}, \mathbf{j}, \mathbf{k}, \mathbf{l} \in[d]^{3},|\mathbf{i}|=\cdots=|\mathbf{k}|=d, \mathbf{e} \in[8]^{3},|\mathbf{e}|=8$.

The following proposition is an important ingredient in the proof of rationality.

Proposition 4.1 For $d=3 n+1$, the projectivization of the linear space

$$
L_{S}=x_{1}^{2 n+3} \cdot \mathbb{C}\left[x_{1}, x_{2}, x_{3}\right]_{n-2} \subset V(d)
$$

is contained in the base scheme $B_{S}$ of the rational map

$$
S_{d}: \quad \mathbb{P} V(d) \rightarrow \mathbb{P} V(4)
$$

with a full triple structure, i.e.

$$
\mathcal{I}_{\mathbb{P}\left(L_{S}\right)}^{3} \supset \mathcal{I}_{B_{S}} .
$$

Similarly, for $d=3 n+2$, the projectivization of the linear space

$$
L_{T}=x_{1}^{2 n+5} \cdot \mathbb{C}\left[x_{1}, x_{2}, x_{3}\right]_{n-3} \subset V(d)
$$

is contained in the base scheme $B_{T}$ of

$$
T_{d}: \quad \mathbb{P} V(d) \rightarrow \mathbb{P} V(8)
$$

with a full triple structure. 
Proof Regardless of whether $d=3 n+1$ or $d=3 n+2$, the conditions that the monomials in (14) or (16) are invariant under the actions of the one-parameter subgroups $\lambda_{H_{1}}$ resp. $\lambda_{H_{2}}$ read

$$
\begin{aligned}
& i_{1}+j_{1}+k_{1}+l_{1}-i_{2}-j_{2}-k_{2}-l_{2}-e_{1}+e_{2}=0, \quad \text { resp. } \\
& i_{2}+j_{2}+k_{2}+l_{2}-i_{3}-j_{3}-k_{3}-l_{3}-e_{2}+e_{3}=0 .
\end{aligned}
$$

Now for $d=3 n+1$ we get

$$
\begin{aligned}
4(3 n+1) & =|\mathbf{i}|+|\mathbf{j}|+|\mathbf{k}|+|\mathbf{l}| \\
& =3\left(i_{2}+j_{2}+k_{2}+l_{2}\right)+\left(e_{1}-e_{2}\right)+\left(e_{3}-e_{2}\right) \\
& =3\left(i_{2}+j_{2}+k_{2}+l_{2}\right)+4-3 e_{2},
\end{aligned}
$$

and for $d=3 n+2$ one has

$$
\begin{aligned}
4(3 n+2) & =|\mathbf{i}|+|\mathbf{j}|+|\mathbf{k}|+|\mathbf{l}| \\
& =3\left(i_{2}+j_{2}+k_{2}+l_{2}\right)+\left(e_{1}-e_{2}\right)+\left(e_{3}-e_{2}\right) \\
& =3\left(i_{2}+j_{2}+k_{2}+l_{2}\right)+8-3 e_{2} .
\end{aligned}
$$

In both cases then it follows that

$$
\begin{aligned}
& i_{1}+j_{1}+k_{1}+l_{1}=4 n+e_{1}, \\
& i_{2}+j_{2}+k_{2}+l_{2}=4 n+e_{2}, \\
& i_{3}+j_{3}+k_{3}+l_{3}=4 n+e_{3} .
\end{aligned}
$$

In particular, for $d=3 n+1, i_{1}+j_{1}+k_{1}+l_{1} \leq 4 n+4$, which means that at most 1 out of the 4 indices $i_{1}, j_{1}, k_{1}, l_{1}$ can be $\geq(4 n+4) / 2+1=2 n+3$. Since $\mathcal{I}_{L_{S}}$ is generated by those $A_{\mathbf{i}}$ with $i_{1}<2 n+3$, this proves the first assertion.

For $d=3 n+2, i_{1}+j_{1}+k_{1}+l_{1} \leq 4 n+8$, whence at most 1 out of $i_{1}, j_{1}, k_{1}, l_{1}$ can be $\geq(4 n+8) / 2+1=2 n+5$, which proves the proposition.

Remark 4.2 By construction, $L_{S}$ (resp. $L_{T}$ ) have the following basic property: For $g \in$ $V(d) \backslash L_{S}$ (resp. $\left.g \in V(d) \backslash L_{T}\right)$, the restriction $\left.S_{d}\right|_{\mathbb{P}\left(L_{S}+\mathbb{C} g\right)}$ (resp. $\left.\left.T_{d}\right|_{\mathbb{P}\left(L_{T}+\mathbb{C} g\right)}\right)$ is linear.

\section{Fiberwise surjectivity of the covariants}

To begin with, we will show how some elements of $L_{S}$ (resp. $L_{T}$ ) can be written as sums of powers. For this let $K$ be a positive integer.

Definition 5.1 Let $\mathbf{b}=\left(b_{1}, \ldots, b_{K}\right) \in \mathbb{C}^{K}$ be given. Then we denote by

$$
p_{i}^{\mathbf{b}}(c):=\prod_{j \neq i, 1 \leq j \leq K} \frac{c-b_{j}}{b_{i}-b_{j}}
$$

for $i=1, \ldots, K$ the interpolation polynomials of degree $K-1$ w.r.t. $\mathbf{b}$ in the one variable $c$. 
Lemma 5.2 Let $\mathbf{b}=\left(b_{1}, \ldots, b_{K}\right) \in \mathbb{C}^{K}, b_{i} \neq b_{j}$ for $i \neq j$, and set $x=x_{1}, y=\lambda x_{2}+\mu x_{3}$, $(\lambda, \mu) \neq(0,0)$. Suppose $d>K$ and put $l_{i}:=b_{i} x+y$. Then for each $c \in \mathbb{C}$ with $c \neq b_{i}, \forall i$,

$$
f(c)=p_{1}^{\mathbf{b}}(c) l_{1}^{d}+\cdots+p_{K}^{\mathbf{b}}(c) l_{K}^{d}-(c x+y)^{d}
$$

is nonzero and divisible by $x^{K}$.

Proof The coefficient of the monomial $x^{A} y^{B}$ in $f(c)$ is equal to

$$
\left(\begin{array}{l}
d \\
A
\end{array}\right)\left(p_{1}^{\mathbf{b}}(c) b_{1}^{A}+\cdots+p_{K}^{\mathbf{b}}(c) b_{K}^{A}-c^{A}\right) .
$$

For $A \leq K-1$ one has

$$
c^{A}=p_{1}^{\mathbf{b}}(c) b_{1}^{A}+\cdots+p_{K}^{\mathbf{b}}(c) b_{K}^{A}
$$

for all $c$ by interpolation.

Choosing $K=2 n+3$, we obtain elements $f(c) \in L_{S}$, and for $K=2 n+5$ elements $f(c) \in L_{T}$. Now for $d=3 n+1$ consider the diagram

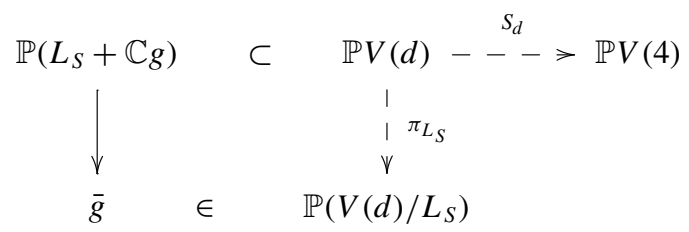

or for $d=3 n+2$ the diagram

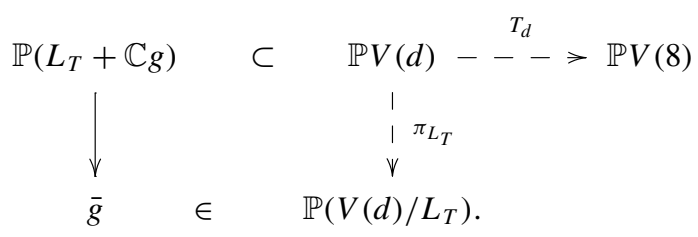

The aim of this section is to prove:

Proposition 5.3 Let $d=3 n+1 \geq 37$. Then there exists a $g \in V(d)$ such that

$$
\left.S_{d}\right|_{\mathbb{P}\left(L_{S}+\mathbb{C} g\right)}: \quad \mathbb{P}\left(L_{S}+\mathbb{C} g\right) \rightarrow \mathbb{P} V(4)
$$

is surjective. For $d=3 n+2 \geq 65$ there exists a $g \in V(d)$ such that

$$
\left.T_{d}\right|_{\mathbb{P}\left(L_{T}+\mathbb{C} g\right)}: \quad \mathbb{P}\left(L_{T}+\mathbb{C} g\right)-\rightarrow \mathbb{P} V(8)
$$

is surjective.

We will prove the case $d=3 n+1$ first. The case $d=3 n+2$ is very similar, and we will deal with it afterwards.

We start by constructing points in the image of $S_{d}$ : 
Lemma 5.4 Consider $S_{d}(f(c)+g)$ as an element of $\mathbb{C}\left[x_{1}, x_{2}, x_{3}, c\right]$ and write

$$
S_{d}(f(c)+g)=Q_{d} c^{d}+\cdots+Q_{0}
$$

with $Q_{i} \in \mathbb{C}\left[x_{1}, x_{2}, x_{3}\right]_{4}$. Then $\left[Q_{i}\right] \in S_{d}\left(\mathbb{P}\left(L_{S}+\mathbb{C} g\right)\right)$ for all $i$.

Proof The map

$$
\begin{aligned}
\varphi: & \mathbb{A}^{1} \rightarrow \mathbb{P} V(4) \\
c & \mapsto S_{d}(f(c)+g)
\end{aligned}
$$

gives a rational curve $X$ in $S_{d}\left(\mathbb{P}\left(L_{S}+\mathbb{C} g\right)\right)$. Since by Remark 4.2, $\left.S_{d}\right|_{\mathbb{P}\left(L_{S}+\mathbb{C g} g\right)}$ is linear, the linear span of $X$ is contained in $S_{d}\left(\mathbb{P}\left(L_{S}+\mathbb{C} g\right)\right)$. Now $\langle X\rangle=\left\langle Q_{0}, \ldots, Q_{d}\right\rangle$ which proves the claim.

Surprisingly, for $i$ large enough, the $Q_{i}$ do not depend on the vector $\mathbf{b}=\left(b_{1}, \ldots, b_{K}\right)$ chosen to construct $f(c)$ :

\section{Proposition 5.5 If}

$$
S_{d}(f(c)+g)=Q_{d} c^{d}+\cdots+Q_{0}, \quad Q_{i} \in \mathbb{C}\left[x_{1}, x_{2}, x_{3}\right]_{4}
$$

and

$$
S_{d}\left(-(c x+y)^{d}+g\right)=Q_{d}^{\prime} c^{d}+\cdots+Q_{0}^{\prime}, \quad Q_{i}^{\prime} \in \mathbb{C}\left[x_{1}, x_{2}, x_{3}\right]_{4},
$$

then $Q_{i}=Q_{i}^{\prime}$ for $i \geq K$.

Proof Write $g$ as a sum of $d$ th powers of linear forms

$$
g=m_{1}^{d}+\cdots+m_{\text {const }}^{d},
$$

where const is a positive integer that will be fixed (independently of $n$ ) in the later discussion. Then (using $\mathcal{I}_{B_{S}} \subset \mathcal{I}_{\mathbb{P}\left(L_{S}\right)}^{3}$ and Lemma 3.1)

$$
\begin{aligned}
S_{d}(f(c)+\epsilon g)= & S_{d}\left(p_{1}^{\mathbf{b}}(c) l_{1}^{d}+\cdots+p_{K}^{\mathbf{b}}(c) l_{K}^{d}-(c x+y)^{d}\right. \\
& \left.+\epsilon m_{1}^{d}+\cdots+\epsilon m_{\text {const }}^{d}\right) \\
= & 24\left(\epsilon^{3} \sum_{\substack{i \\
j<k<p}} p_{i}^{\mathbf{b}}(c) I\left(l_{i}, m_{j}, m_{k}, m_{p}\right)^{n} l_{i} m_{j} m_{k} m_{p}\right. \\
& -\epsilon^{3} \sum_{j<k<p} I\left(c x+y, m_{j}, m_{k}, m_{p}\right)^{n}(c x+y) m_{j} m_{k} m_{p} \\
& \left.+\epsilon^{4} \sum_{i<j<k<p} I\left(m_{i}, m_{j}, m_{k}, m_{p}\right)^{n} m_{i} m_{j} m_{k} m_{p}\right)
\end{aligned}
$$

For $\epsilon=1$ we find

$$
S_{d}(f(c)+g)=\sum_{i, j, k, p} p_{i}^{\mathbf{b}}(c) I\left(l_{i}, m_{j}, m_{k}, m_{p}\right)^{n} l_{i} m_{j} m_{k} m_{p}+S_{d}\left(-(c x+y)^{d}+g\right) .
$$


Since $\operatorname{deg} p_{i}^{\mathbf{b}}=K-1$, the assertion follows.

Next we will investigate the dependence of $Q_{t}$ on $n$ for $t \geq K$. We choose a fixed constant $\wp \in \mathbb{N}$ (the "precision") with $d-\wp+1 \geq K$ and $\wp \leq n$ (later $\wp$ will be a prime number).

Lemma 5.6 For $3 n+1-\wp \leq s \leq 3 n$, the coefficient of $c^{s}$ in $I\left(c x+y, m_{i}, m_{j}, m_{k}\right)^{n}$ is of the form

$$
\varrho^{n} P(n)
$$

where $\varrho \in \mathbb{C}$ (independent of $n$ and $s$ ) and $P(n)$ is a polynomial of degree $3 n-s<\wp . P(n)$ is, as a polynomial in $\mathbb{C}[n]$ divisible by

$$
\left(\begin{array}{c}
n \\
n-\left\lceil\frac{s}{3}\right\rceil
\end{array}\right)
$$

If the coefficients of the $m_{i}$ are integers and $\wp$ is a prime number, then the reduction of the coefficient of $c^{s}$ in $I\left(c x+y, m_{i}, m_{j}, m_{k}\right)^{n}$ modulo $\wp$ is still of the form

$$
\varrho^{n} P(n)
$$

with $\varrho \in \mathbb{F}_{\wp}$ and $P(n) \in \mathbb{F}_{\wp}[n]$ satisfying the same independence and divisibility conditions as above.

Proof Calculating either over $\mathbb{C}$ or over $\mathbb{F}_{\wp}$, we have

$$
\begin{aligned}
& I\left(c x+y, m_{i}, m_{j}, m_{k}\right)^{n} \\
& \quad=\left(c x+y, m_{i}, m_{k}\right)^{n}\left(c x+y, m_{i}, m_{j}\right)^{n}\left(c x+y, m_{j}, m_{k}\right)^{n}\left(m_{i}, m_{j}, m_{k}\right)^{n} \\
& \quad=\left(\xi_{i k} c+\eta_{i k}\right)^{n}\left(\xi_{i j} c+\eta_{i j}\right)^{n}\left(\xi_{j k} c+\eta_{j k}\right)^{n}\left(m_{i}, m_{j}, m_{k}\right)^{n}
\end{aligned}
$$

where the $\xi$ 's and $\eta$ 's are constants (fixed once the $m$ 's are fixed). If any of the $\xi$ 's vanishes the polynomial $I\left(c x+y, m_{i}, m_{j}, m_{k}\right)^{n}$ is of degree $\leq 2 n$ in $c$. Since $s>3 n-\wp \geq 2 n$, in this situation the coefficient of $c^{s}$ is 0 and we are finished. Assume therefore that the $\xi$ 's are invertible.

The above expression expands to

$$
\left(\sum_{p=1}^{n}\left(\begin{array}{l}
n \\
p
\end{array}\right) \xi_{i k}^{p} c^{p} \eta_{i k}^{n-p}\right) \cdot\left(\sum_{q=1}^{n}\left(\begin{array}{l}
n \\
q
\end{array}\right) \xi_{i j}^{q} c^{q} \eta_{i j}^{n-q}\right) \cdot\left(\sum_{r=1}^{n}\left(\begin{array}{l}
n \\
r
\end{array}\right) \xi_{j k}^{r} c^{r} \eta_{j k}^{n-r}\right) \cdot\left(m_{i}, m_{j}, m_{k}\right)^{n}
$$

and the coefficient of $c^{s}$ is

$$
\left(m_{i}, m_{j}, m_{k}\right)^{n} \sum_{p+q+r=s}\left(\begin{array}{l}
n \\
p
\end{array}\right)\left(\begin{array}{l}
n \\
q
\end{array}\right)\left(\begin{array}{l}
n \\
r
\end{array}\right) \xi_{i k}^{p} \xi_{i j}^{q} \xi_{j k}^{r} \eta_{i k}^{n-p} \eta_{i j}^{n-q} \eta_{j k}^{n-r} .
$$

Put $p^{\prime}=n-p, q^{\prime}=n-q, r^{\prime}=n-r$ and rewrite this as

$$
\left(\left(m_{i}, m_{j}, m_{k}\right) \xi_{i k} \xi_{i j} \xi_{j k}\right)^{n} \sum_{p^{\prime}+q^{\prime}+r^{\prime}=3 n-s}\left(\begin{array}{c}
n \\
p^{\prime}
\end{array}\right)\left(\begin{array}{c}
n \\
q^{\prime}
\end{array}\right)\left(\begin{array}{c}
n \\
r^{\prime}
\end{array}\right) \xi_{i k}^{-p^{\prime}} \xi_{i j}^{-q^{\prime}} \xi_{j k}^{-r^{\prime}} \eta_{i k}^{p^{\prime}} \eta_{i j}^{q^{\prime}} \eta_{j k}^{r^{\prime}} .
$$


The first claim of the lemma over $\mathbb{C}$ is obvious now. The reductions of the binomial coefficients modulo $\wp$ are polynomials in $n$ over $\mathbb{F}_{\wp}$ if $p^{\prime}, q^{\prime}, r^{\prime}<\wp$. Our conditions on $s$ imply this, since

$$
p^{\prime}, q^{\prime}, r^{\prime} \leq p^{\prime}+q^{\prime}+r^{\prime}=3 n-s<\wp .
$$

As for the stated divisibility property in $\mathbb{C}[n]$ and $\mathbb{F}_{\wp}[n]$, remark that in

$$
\left(\begin{array}{l}
n \\
p^{\prime}
\end{array}\right)\left(\begin{array}{l}
n \\
q^{\prime}
\end{array}\right)\left(\begin{array}{l}
n \\
r^{\prime}
\end{array}\right)
$$

with $p^{\prime}+q^{\prime}+r^{\prime}=3 n-s$, at least one of $p^{\prime}, q^{\prime}, q^{\prime}$ is $\geq n-\left\lceil\frac{s}{3}\right\rceil$.

Proposition 5.7 For $d-\wp+1 \leq t \leq d$, the coefficient of each monomial $\mathbf{x}^{\mathrm{i}}$ in $Q_{t}$ is of the form

$$
\sum_{\nu=1}^{\left(\begin{array}{c}
\text { const } \\
3
\end{array}\right)} \varrho_{v}^{n} P_{\nu}(n)
$$

where $\varrho_{v} \in \mathbb{C}$ are constants (independent of $n$ ), and $P_{v}(n)$ are polynomials of degree $\leq d-t<\wp$, which are divisible by

$$
\left(\begin{array}{c}
n \\
n-\left\lceil\frac{t}{3}\right\rceil
\end{array}\right)
$$

If $g$ can be written as sum of powers with integer coefficients and $\wp$ is a prime number, the same is true for the reduction of $Q_{t} \bmod \wp$.

Proof $Q_{t}$ is the coefficient of $c^{t}$ in

$$
\text { (-24) } \sum_{1 \leq i<j<k \leq \mathrm{const}} I\left(c x+y, m_{i}, m_{j}, m_{k}\right)^{n}(c x+y) m_{i} m_{j} m_{k}
$$

(cf. (29)), so we may apply Lemma 5.6 with $s=t$ and $s=t-1$.

Definition 5.8 For $d-\wp+1 \leq t \leq d$, we put

$$
R_{t}:=\frac{Q_{t}}{\left(\begin{array}{c}
n \\
n-\left\lceil\frac{t}{3}\right\rceil
\end{array}\right)} .
$$

Proof of Proposition 5.3 (For $d=3 n+1$ ) Let const $=9$ and consider

$$
g=m_{1}^{d}+\cdots+m_{\text {const }}^{d}
$$

with

$$
\begin{array}{lll}
m_{1}=x_{1}+3 x_{2}+9 x_{3} & m_{4}=x_{1}+6 x_{2}-10 x_{3} & m_{7}=-3 x_{2}+2 x_{3} \\
m_{2}=-10 x_{1}+x_{2}+4 x_{3} & m_{5}=4 x_{1}-8 x_{2}-10 x_{3} & m_{8}=8 x_{1}-4 x_{2}-4 x_{3} \\
m_{3}=8 x_{1}+4 x_{2}+6 x_{3} & m_{6}=-3 x_{1}+7 x_{2}-4 x_{3} & m_{9}=-10 x_{1}+4 x_{2}+6 x_{3} .
\end{array}
$$


For $\wp=11$ we perform our construction with $x=x_{1}$ and two different values for $y$, namely $y_{1}=x_{2}$ and $y_{2}=x_{3}$. We obtain 22 quartics $R_{d}^{y_{1}}, \ldots, R_{d-10}^{y_{1}}, R_{d}^{y_{2}}, \ldots, R_{d-10}^{y_{2}}$. By Lemma 5.4 and Proposition 5.5 these quartics are in the image of $\left.S_{d}\right|_{\mathbb{P}\left(L_{S}+\mathbb{C g}\right)}$ if

$$
d-10 \geq K \quad \Longleftrightarrow \quad 3 n+1-10 \geq 2 n+3 \quad \Longleftrightarrow \quad n \geq 12 .
$$

The coefficients of the $R_{j}^{y_{i}}$ form a $15 \times 22$ matrix $M(n)$ with entries of the form $\sum_{\nu=1}^{84} \varrho_{v}^{n} P_{v}(n)$ by Proposition 5.7. Modulo 11 this matrix becomes periodic in $n$ with period $11 \cdot 10=110$. With a computer algebra program it is straightforward to check that all these matrices have full rank 15. A Macaulay2 script doing this can be found at [7]. This proves the claim for $d=3 n+1$.

Let us turn to the case $d=3 n+2$. The whole procedure is similar in this case. If we take $K=2 n+5$ Lemma 5.2, Proposition 5.5, Lemma 5.6 and Proposition 5.7 remain true as stated and Definition 5.8 still makes sense.

Proof of Proposition 5.3 (For $d=3 n+2$ ) Let const $=9$ and consider

$$
g=m_{1}^{d}+\cdots+m_{\text {const }}^{d}
$$

with $m_{i}$ as above.

For $\wp=19$ we perform our construction with $x=x_{1}$ and three different values for $y$, namely $y_{1}=x_{2}, y_{2}=x_{3}$ and $y_{3}=x_{2}+x_{3}$. We obtain 57 octics $R_{d}^{y_{1}}, \ldots, R_{d-18}^{y_{1}}, R_{d}^{y_{2}}, \ldots$, $R_{d-18}^{y_{2}}, R_{d}^{y_{3}}, \ldots, R_{d-18}^{y_{3}}$. By Lemma 5.4 and Proposition 5.5 these octics are in the image of $\left.S_{d}\right|_{\mathbb{P}\left(L_{T}+\mathbb{C} g\right)}$ if

$$
d-18 \geq K \quad \Longleftrightarrow \quad 3 n+2-18 \geq 2 n+5 \quad \Longleftrightarrow \quad n \geq 21 .
$$

The coefficients of the $R_{j}^{y_{i}}$ from a $45 \times 57$ matrix $M(n)$ with entries of the form $\sum_{v=1}^{84} \varrho_{v}^{n} P_{v}(n)$ by Proposition 5.7. Modulo 19 this matrix becomes periodic in $n$ with period $19 \cdot 18=342$. With a computer algebra program it is straightforward to check that all these matrices have full rank 45. A Macaulay2 script doing this can be found in [7]. This proves the claim for $d=3 n+2$.

\section{Sections of principal bundles and proof of rationality}

We will now show how to conclude the proof in the case $d=3 n+1$. We make some comments on the case $d=3 n+2$ when they are in order, but otherwise leave the obvious modifications to the reader. Let $(\mathbb{P} V(4))_{\mathrm{vs}} \subset \mathbb{P} V(4)$ be the open subset of very stable points with respect to the action of $\bar{G}$ and the $\bar{G}$-linearized line bundle $\mathcal{O}(3)$ (very stable means stable with trivial stabilizer). Now the essential point is:

Proposition 6.1 The quotient morphism

$$
(\mathbb{P} V(4))_{\mathrm{vs}} \rightarrow(\mathbb{P} V(4))_{\mathrm{vs}} / \bar{G}
$$

is a principal $\bar{G}$-bundle in the Zariski topology. 
Proof See [22], Proposition 2. This holds also true with $V(4)$ replaced with $V(8)$.

It follows that this $\bar{G}$-bundle has a section defined generically which we will denote by $\sigma_{4}$.

Proof of Theorem 1.1 Consider the graph

$$
X=\left\{(g, \bar{g}, f) \mid \pi_{L_{S}}(g)=\bar{g}, S_{d}(g)=f\right\} \subset \mathbb{P} V(d) \times \mathbb{P}\left(V(d) / L_{S}\right) \times \mathbb{P} V(4)
$$

and the diagram

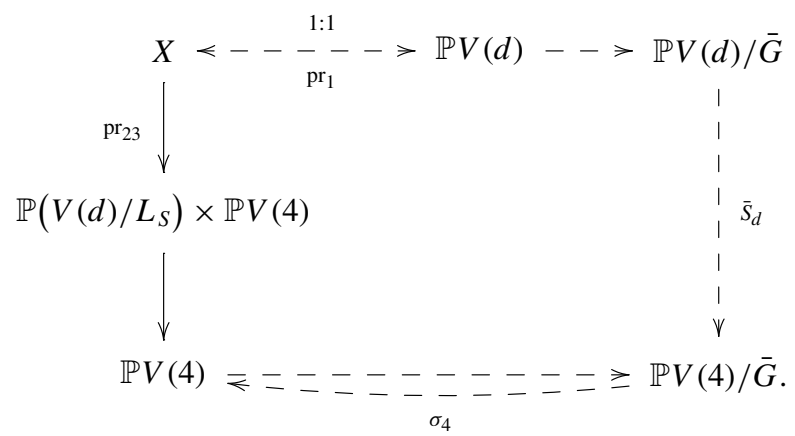

By Proposition 5.3 the projection $\mathrm{pr}_{23}$ is dominant. It follows then from Remark 4.2 that $X$ is birational to a vector bundle over $\mathbb{P}\left(V(d) / L_{S}\right) \times \mathbb{P} V(4)$ and hence also over $\mathbb{P} V(4)$. After replacing $\sigma_{4}$ by a translate, we can assume that $\sigma_{4}$ meets an open set $U \subset \mathbb{P} V(4)$ over which this vector bundle is trivial. Since $\bar{G}$ acts generically freely on $\mathbb{P} V(4)$, we can pull back the above vector bundle structure via $\sigma_{4}$ and obtain that $\mathbb{P} V(d) / \bar{G}$ is birational to $\mathbb{P} V(4) / \bar{G} \times \mathbb{P}^{N}$ with $N=\operatorname{dim} V(d)-\operatorname{dim} V(4)$. If $d \geq 37$ as in Proposition 5.3, then certainly $N \geq 8$ and since $(\mathbb{P} V(4) / \bar{G}) \times \mathbb{P}^{8}$ is rational, $\mathbb{P} V(d) / \bar{G}$ is rational. In the case $d=3 n+2$ the same argument works since the space of octics is also stably rational of level 8. This proves Theorem 1.1.

Acknowledgements We thank the referee for pointing out that the arguments of our proof of Thorem 1.1 do not only show rationality over $\mathbb{C}$ but even over $\mathbb{Q}$. We would like to thank Fedor Bogomolov and Yuri Tschinkel for suggesting this problem and helpful discussions.

Open Access This article is distributed under the terms of the Creative Commons Attribution Noncommercial License which permits any noncommercial use, distribution, and reproduction in any medium, provided the original author(s) and source are credited.

\section{References}

1. Artin, M., Mumford, D.: Some elementary examples of unirational varieties which are not rational. Proc. Lond. Math. Soc. 25(3), 75-95 (1972)

2. Beauville, A., Colliot-Thélène, J.-L., Sansuc, J.-J., Swinnerton-Dyer, S.P.: Variétés stablement rationnelles et non-rationnelles. Ann. Math. 121, 283-318 (1985)

3. Beklemishev, N.: Invariants of cubic forms in four variables. Vestnik Moscov. Univ. Ser. I Mat. Mekh. 2, 42-49 (1982)

4. Bogomolov, F.: Stable rationality of quotient varieties by simply connected groups. Mat. Sbornik 130, 3-17 (1986) 
5. Bogomolov, F.: Rationality of the moduli of hyperelliptic curves of arbitrary genus. In: Conference on Algebraic Geometry, Vancouver, 1984. CMS Conference Proceedings, vol. 6, pp. 17-37. Amer. Math. Soc., Providence (1986)

6. Bogomolov, F., Katsylo, P.: Rationality of some quotient varieties. Mat. Sbornik 126, 584-589 (1985)

7. Böhning, C., Graf von Bothmer, H.-C.: Macaulay2 scripts to check the surjectivity of the Scorza and Octa maps. Available at http://www.uni-math.gwdg.de/bothmer/rationality (2008)

8. Clemens, H., Griffiths, P.: The intermediate Jacobian of the cubic threefold. Ann. Math. 95, 281-356 (1972)

9. Colliot-Thélène, J.-L., Sansuc, J.-J.: The rationality problem for fields of invariants under linear algebraic groups (with special regards to the Brauer group). In: Algebraic Groups and Homogeneous Spaces. Tata Institute of Fundamental Research Studies in Mathematics, pp. 113-186. Tata Inst. Fund. Res., Mumbai (2007)

10. Dolgachev, I.: Rationality of fields of invariants. In: Proceedings of Symposia in Pure Mathematics, vol. 46, pp. 3-16 (1987)

11. Dolgachev, I.: Lectures on Invariant Theory. London Mathematical Society Lecture Note Series, vol. 296. Cambridge Univ. Press, Cambridge (2003)

12. Dolgachev, I.: Topics in classical algebraic geometry. part I. Available at http://www.math.lsa.umich. edu/ idolga/lecturenotes.html

13. Dolgachev, I., Kanev, V.: Polar covariants of plane cubics and quartics. Adv. Math. 98, 216-301 (1993)

14. Grace, J.H., Young, W.H.: The Algebra of Invariants. Cambridge Univ. Press, Cambridge (1903). Reprinted by Chelsea, New York (1965)

15. Iskovskikh, V.A., Prokhorov, Yu.G.: Fano Varieties. In: Parshin, A.N., Shafarevich, I.R. (eds.) Algebraic Geometry V. Encyclopedia of Mathematical Sciences, vol. 47. Springer, Berlin/Heidelberg (1999)

16. Katsylo, P.I.: Rationality of the moduli spaces of hyperelliptic curves. Izv. Akad. Nauk SSSR Ser. Mat. 48, 705-710 (1984)

17. Katsylo, P.I.: Rationality of moduli varieties of plane curves of degree $3 k$. Math. USSR Sbornik 64(2) (1989)

18. Katsylo, P.I.: On the birational geometry of the space of ternary quartics. Adv. Sov. Math. 8, 95-103 (1992)

19. Katsylo, P.I.: Rationality of the moduli variety of curves of genus 3. Comment. Math. Helvetici 71, 507-524 (1996)

20. Ranestad, K., Schreyer, F.: Varieties of sums of powers. J. Reine Angew. Math. 525, 147-181 (2000)

21. Saltman, D.: Noether's problem over an algebraically closed field. Invent. Math. 77, 71-84 (1984)

22. Shepherd-Barron, N.I.: The rationality of some moduli spaces of plane curves. Compos. Math. 67, 51-88 (1988) 